\title{
Changing Trends of Antibiotic Susceptibility Pattern among Vibrio cholerae 01 Serovars from Sporadic Cases in Coastal Karnataka, South India
}

\author{
Mamatha Ballal $^{1,2}$, Suganthi Martena Devadas², Nishanth Bhat ${ }^{1}$ \\ Department of Microbiology, Melaka Manipal Medical College, Manipal University, Manipal. ${ }^{\prime}$ \\ Enteric Pathogens Lab, Manipal University, Manipal ${ }^{2}$
}

\begin{abstract}
Changing patterns of antibiotic sensitivity of $V$. cholerae is a major concern as Multiple-Antibiotic-Resistant (MAR) $V$. cholerae isolates are being reported from various parts of the world. The study was conducted to analyze the prevalence and antibiotic resistance pattern of V. cholerae O1. Samples were processed according to the standard guidelines. Sporadic outbreaks occurred in June 2004 and March 2010. Antibiotic resistance was seen in the strains isolated. A large number of cholera epidemics have been associated with MAR strains of $V$. cholerae. This makes it imperative that all isolates be constantly subjected to susceptibility testing and resistance patterns to each antibiotic be monitored.
\end{abstract}

Keywords: Aeromonas hydrophila, Vibrio cholerae Inaba, Vibrio cholerae Ogawa, Tetracycline

\section{INTRODUCTION}

Vibrio cholerae is one of the major etiological agents causing watery diarrhoea in almost all parts of the society, especially in the lower socio economic strata of developing countries like India. Sporadic or epidemic cases of cholera are reported frequently from both adults and children alike. Epidemics of cholera caused by toxigenic V.cholerae $\mathrm{O} 1$ and V.cholerae $\mathrm{O} 139$ represent a major public health problem ${ }^{1}$ for India with frequent epidemic potential as an important cause of morbidity and mortality. The use of antimicrobial agents in cholera is generally accepted as a method of reducing the duration and volume of diarrhea as well as decreasing the period of Vibrio cholerae excretion in stool. ${ }^{2}$

\section{MATERIALSAND METHODS}

Fecal specimens from patients with acute or watery diarrhea during the sporadic outbreak were received for microscopy and culture of routine enteric pathogens at

\section{Corresponding Author :}

Dr. Mamatha Ballal, Phd FIMSA

Professor of Microbiology (MMMC),

Enteric Pathogens Lab,

Manipal University, Manipal.

Karnataka, India. 576104

E mail: mamatha_98@yahoo.com
Diagnostic Microbiology Laboratory, during June 2004 and March 2010. Sixty and 92 stool specimens were collected before antibiotic administration and processed for identification of various pathogens respectively. No transport medium was employed as fresh specimen was received in the laboratory within 30 minutes and processed immediately without any further delay. Routine stool microscopy of saline and iodine preparations was examined for intestinal parasites. A battery of culture media - Alkaline Peptone Water, Selenite Feces broth, Blood agar, Mac Conkey agar, Thiosulphate Citrate Bile salt Sucrose agar (TCBS) were used for the isolation of bacterial pathogens. Bacteriological analysis was done by standard laboratory techniques. ${ }^{3,4}$ Vibrio cholera was serotyped by slide agglutination test using specific antisera (Difco, USA and NICED, Kolkata). The antimicrobial susceptibility testing was performed for $V$. cholerae by using the Kirby Bauer disc diffusion technique using commercially available discs (Hi-media). The antibiotics used were ampicillin (AMP $10 \mu \mathrm{g}$ ), chloramphenicol (CHL $30 \mu \mathrm{g}$ ), tetracycline (TET 30 $\mu \mathrm{g}$ ), cotrimoxazole (SXT $25 \mu \mathrm{g}$ ), ciprofloxacin (CIP 5 $\mu \mathrm{g}$ ), nalidixic acid (NAL $30 \mu \mathrm{g}$ ) and gentamicin (GEN $10 \mu \mathrm{g}$ ). The current CLSI guidelines were followed for interpretation of the results and E.coli ATCC 25922 was used as the control strain.

Patients' clinico-demographic details which include age, 
sex, ward / OPD, presenting clinical history and underlying illness were noted wherever applicable. Institutional Ethical Committee approval was obtained.

\section{RESULTS}

An outbreak of cholera occurred in the coastal town, in the month of June, 2004. A total of 100 patients were admitted to our tertiary care hospital with the presentation of acute watery diarrhea with moderate to severe dehydration. Out of the total patients, 60 stool samples were received in the laboratory for the identification of the causative agent, of which 36 (60\%) stool samples were found to be positive for V.cholerae. Thirty three $(91.6 \%)$ samples were positive for the biotype El Tor serotype Ogawa and 3 (8.3\%) samples were positive for serotype Inaba. Another sporadic outbreak occurred during March to June, 2010. A total of 92 stool samples were processed in the laboratory, of which 11 samples $(11.95 \%)$ were found to be positive as Vibrio cholera O1 biotype El Tor serotype Ogawa.

The analysis of antimicrobial resistance in 2004 and 2010 as shown in Figure I makes it evident that there is a change in the resistance pattern of the isolates. In 2010 there was increased resistance of the Vibrio cholera isolates towards TET, SXT \& AMP, however there was a decreased resistance towards CHL, CIP \& GEN.

\section{DISCUSSION}

V.cholerae O1 Inaba existed for a brief period in 2004 and was replaced by $V$. cholerae O1 Ogawa in the subsequent years. Vibrio cholerae Ogawa was the

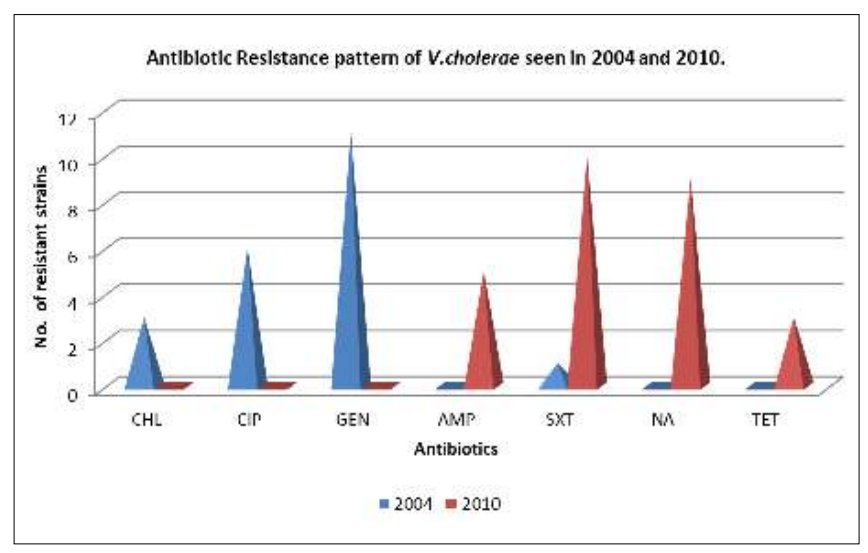

Fig. 1: Graphical representation of antibiotic resistance pattern of V.cholerae O1 seen in 2004 and 2010. predominant isolate in 2004 as well as in 2010. During the last decade, the dominance of Ogawa belonging to the $\mathrm{O} 1$ serotype has seen a shift to Inaba in the last few years of the decade as cases reported from Chandigarh, Bangladesh and Vellore. ${ }^{2,5,6}$

Changing patterns of antibiotic sensitivity of V.cholerae is a major concern as multiple antibiotic resistant V.cholerae isolates are being reported from various parts of the world. ${ }^{5,-9}$ In the present correspondence, we report the appearance of tetracycline resistance in V.cholerae O1 biotype El Tor serovar Ogawa isolated in this coastal region of India. The observation has epidemiological and clinical importance as tetracycline has been the main stay of antibiotic treatment for cholera. In India, tetracycline resistance has been reported in Inaba serovar but sparsely reported in Ogawa serovar. ${ }^{5,6}$

It is important to note that tetracycline-resistant V.cholerae $\mathrm{O} 1 \mathrm{Ogawa}$ isolates are being reported from Madasgar, Bangladesh, Tanzania, Zaire, Latin America and India (Southern and Eastern regions). The pattern of shift in antibiotic resistance indicates an enhanced mobility in genetic elements, which confer resistance to antibiotics. Recently genetic elements, such as a class I integrin and SXT constin carrying tetracyclineresistant genes, tet $\mathrm{G}$ and tetA respectively have also been reported to be associated with the spread of genetic determinants of resistance to antimicrobial agents. ${ }^{9,10}$

The extensive use of tetracycline / doxycycline might have led to the rapid emergence and spread of tetracycline-resistant isolates of Vibrio. The molecular mechanism of tetracycline resistance in Vibrio must be studied and the transfer of such resistant genes has to be epidemiologically monitored.

To conclude, a large number of cholera epidemics have been associated with multiple-antibiotic-resistant (MAR) strains of V.cholerae. ${ }^{11}$ A sudden increase in antibiotic resistance as reported here cannot be ignored; future epidemics in the MAR (Multiple Antibiotic Resistance) serotypes may be imminent. This makes it imperative that all isolates be constantly subjected to susceptibility testing and resistance patterns to each antibiotic to be monitored. Thus antibiotics should be judiciously used in the management of diarrheal diseases. The importance of reporting all cases should be emphasized, with the ultimate goal being a thorough 
understanding of the constantly changing and obscure epidemiology and resistance patterns of V. cholera.

\section{Conflict of Interest: None}

\section{REFERENCES}

1. Das S, Choudhry S, Saha R, Vishnampettai GR, Kaur K, Sarkar BL. Emergence of multiple drug resistance Vibrio cholera $\mathrm{O} 1$ in East India. J Infect Dev Ctries 2011;5(4):294-8.

2. Taneja N, Samanta P, Mishra A, Sharma M. Emergence of tetracycline resistance in Vibrio cholerae O1 biotype El Tor serotype Ogawa from north India. Indian J Pathol Microbiol 2010;53(4):865-6.

3. Cheesebrough M. Examination of fecal specimens. District laboratory practices in tropical countries. Part 2. 2nd edition. Cambridge University Press;p. 97-105.

4. WHO manual. Available at http://www.antimicrobialresistance. dk/data/images/protocols/gfn_biochem_final.pdf.

5. Roy CA, Pan A, Dutta D, Muhkopadhyay AK, Ramamurthy T, Nandy RK, et al. Emergence of tetracycline - resistant Vibrio cholerae O1 serotypes Inaba, in Kolkata, India. Jpn J Infect Dis 2010;61:128-9.
6. Jesudason MV. Change in serotype and appearance of tetracycline resistance in $V$. cholerae $\mathrm{O} 1$ in Vellore, south India. Indian J Med Microbiol 2006:152-3.

7. Bradley SR, Rahman M, Yunus M, Khan EH. Antimicrobial Resistance in organisms causing diarrheal disease. Clin Infect Dis 1997;24:102-5.

8. Jacques-Albert D, Olivat RA, Davidra R, Migliani R, Ranjalahy J, Mauclere P. Emergence and rapid spread of tetracycline - resistant Vibrio cholerae strains, Madagascar. Emerg Infect Dis 2002;8:336-8.

9. Iwanaga M, Toma C, Miyazato T, Insisiengmay S, Nakasone N, Ehara M. Antibiotic resistance conferred by a class I integrin and SXT constin in Vibrio cholerae O1 strains isolated in Laos. Antimicrob Agents Chemother 2004;48: 2364-9.

10. Ceccarelli D, Salvia AM, Sami J, Cappuccinelli P, Colombo MM. New cluster of plasmid located class 1 integrons in Vibrio cholerae $\mathrm{O} 1$ and a dfrA 15 cassette-containing integrin in Vibrio parahemolyticus isolated in Angola. Antimicrob Agents Chemother 2006;50:2493-9.

11. Shah M, Faruque M, Johirul I, Qazi SA, Kuntal B, Faruque ASG, Nair GB, et al. An improved technique for isolation of environmental Vibrio cholerae with epidemic potential: Monitoring the emergence of a multiple-antibiotic-resistant epidemic strain in Bangladesh. J Infect Dis 2006;193:1029-36. 